\title{
X-ray Microtomographic Analysis of $\alpha$ Tricalcium Phosphate-Poly(lactic-co-glycolic) Acid Nanocomposite Degradation
}

\author{
C.E. Barrett,; R.E. Cameron
}

Cambridge Centre For Medical Materials, Department of Materials Science and Metallurgy, University of Cambridge, 27 Charles Babbage Road, Cambridge, UK, CB3 0FS

\section{Abstract}

The degradation characteristics of $\alpha$ TCP-PLGA(50:50) nanocomposites containing varying ceramic weight loadings in an aqueous medium have been assessed using $\mathrm{X}$ ray microtomography $(\mathrm{X} \mu \mathrm{T})$. Also measured were bulk density changes, pharmaceutic drug release and medium acidification for the degrading materials.

Calcium phosphate addition to the polymer leads to increasing delays in the onset of degradation medium acidification and tetracycline release. Bulk density changes with time for all composite materials measured using a buoyancy method were well described during the initial degradation regime by a $t^{\frac{1}{2}}$ function. PLGA density evolution follows a linear function of time which indicates a differing water absorption process occurring in the pure polymer compared with the nanocomposites.

Nanocomposite microtomographic analysis over the same period elucidated a core-periphery structure caused by water imbibition. Peripheral regions closest to the specimen surface exhibit reduced attenuation coefficients compared with the core which may be characteristic of a frontal system caused by a polymer phase transition. The front position and specimen swelling are adequately described by a $t^{\frac{1}{2}}$ and complementary error function respectively which if assessed under the assumption of a diffusion controlled process yields a diffusion coefficient of water in all nanocomposites at $37^{\circ} \mathrm{C}$ of $4.8 \times 10^{-14} \mathrm{~cm}^{2} \mathrm{~s}^{-1}$. Nevertheless, a $\sqrt{t}$ dependence is a necessary but not sufficient condition of a Fickian diffusion process. For all nanocomposite types both $\mathrm{X} \mu \mathrm{T}$ data and bulk density measurements exhibited no variations with ceramic filler content.

Keywords: Poly(lactic-co-glycolic) acid, Calcium phosphate, Nanocomposite, X-ray microtomography, Degradation kinetics

\footnotetext{
*Corresponding author, e_mail: cbaz577@gmail.com, tel: (0044) 07963011934
}

\section{Introduction}

Composites composed of nanoparticulate $\alpha$ Tricalcium phosphate evenly dispersed in poly(lactic-co-glycolic) acid (50:50 monomer ratio) are candidate materials for use in resorbable orthopaedic devices.

The investigation of bioceramic nanocomposites is motivated by the weight of evidence supporting the biological and physical enhancement of biodegradable polymers by nanoparticulate incorporation compared with equivalent microcomposites. Properties enhanced by nanoparticle addition include greater material moduli, suppression of autocatalytic degradation and cytotoxicity by acid degradation product neutralization. A review of the rationale and evidence for the aforementioned improvements to PLGA properties by $\alpha$ TCP incorporation was performed by Barrett et al. [1]. Additionally, preliminary evaluations of $\alpha$ TCP-PLGA nanocomposite physical property changes with degradation time may be found in the works of Yang et al.[2] and Ehrenfried et al.[3] with further complementary biological characterization of the degrading materials by Meyer et al. [4].

The absence of systematization in the assessment of degradable polymer-ceramic systems has been identified by Vert [5] as a salient problem in modern biodegradable material investigation which hinders the comparison of material behaviour between researchers. Moreover, material modellers often cite the dearth of quantitative polymer degradation and erosion rate measurements as major impediment to predictions of biodegradable device performance and refinement of such models to better predict device behaviour in vivo(author?) [6]. The principal impediment to biomaterial degradation property evaluation is the small length scales associated with degradation phenomena which limits the effectiveness of conventional characterization methods.

This article details characterization of the degradation behaviour of pure PLGA(50:50) and $\alpha \mathrm{TCP}-$ PLGA(50:50) containing 10, 20, 30 and $40 \mathrm{wt} \% \alpha \mathrm{TCP}$ nanoparticles which is a substantial step in rationalizing biodegradable material characterization. X-ray microtomographic analysis is used to evaluate internal morphological changes as functions of degradation time to assess the effects of water imbibition which is a major factor in the evolution of material properties. Thus far most studies of pure PLGA or polymer-ceramic composite de- 
gradation have evaluated only specimen bulk changes. Information regarding internal composite morphological changes therefore is a substantial improvement in the knowledge of these materials which will aid material rationalization and optimization of devices incorporating the material.

In parallel with X-ray microtomography, degradation medium acidification measurements, tetracycline drug release data and specimen bulk density changes measured using a buoyancy technique are presented to systematically elucidate the degradation kinetics of the nanocomposite materials.

\section{Sample preparation}

\subsection{Materials}

Nanoparticle $\alpha \mathrm{TCP}$ was synthesized at room temperature using an aqueous precipitation reaction according to

$$
\begin{aligned}
& 2 \mathrm{H}_{3} \mathrm{PO}_{4\{\mathrm{aq}\}}+3 \mathrm{Ca}(\mathrm{OH})_{2\{\mathrm{aq}\}} \rightarrow \\
& \qquad \mathrm{Ca}_{3}\left(\mathrm{PO}_{4}\right)_{2\{\mathrm{aq}\}}+6 \mathrm{H}_{2} \mathrm{O} .
\end{aligned}
$$

Aqueous $0.187 \mathrm{M}$ calcium hydroxide (Sigma Aldrich, UK) and $0.121 \mathrm{M}$ phosphoric acid (analytical reagent grade, BDH Laboratory Supplies, UK) were stirred separately for one hour then mixed together and stirred for a further hour in order to homogenize the solution. The solution was left to age for 24 hours then filtered leaving a gelatinous paste. After evaporating the water in a drying cabinet the solid was crushed with a mortar and pestle and ball milled for four hours. The dried powder was sieved using a $75 \mu \mathrm{m}$ mesh followed by sintering at $1400^{\circ} \mathrm{C}$ for four hours after which the sample was cooled rapidly to room temperature.

TCP polymorph phase identification was performed using an X-ray powder diffractometer (Philips X-Pert PW3020 vertical diffractometer) employing $\mathrm{Cu} \mathrm{K} \alpha$ Xrays operating at source voltage and current settings of $40 \mathrm{kV}$ and $40 \mathrm{~mA}$. Diffraction patterns were acquired over the angle range $10^{\circ}-50^{\circ}$ using a step size of $0.02^{\circ}$ and dwell time of 30 seconds.

Post-acquisition, quantitative peak fitting was performed using the software Highscore Plus employing the reference intensity ratio method (RIR) to assess the material phase content. Acquired powder diffraction patterns were matched to material reference diffraction data from the International Centre for Diffraction Data (ICDD), selecting the patterns corresponding to $\alpha, \beta \mathrm{TCP}$, and $\mathrm{HA}$ in addition to other commonly occurring compounds in calcined materials such as calcium oxide. The RIR method determined that the phase purity of the TCP was $>95 \%$ which meets the required criterion for polymorph phase purity.
The $\alpha \mathrm{TCP}$ crystal size was assessed using peak broadening analysis of X-rays diffracted from a crystal of width $d$ using the same Phillips diffractometer system according to the Scherrer formula

$$
d=\frac{K \lambda}{\gamma \cos (2 \theta)}
$$

where $\gamma$ denotes the crystal diffraction peak full-width half maximum, $K$ is a geometrical constant, $\lambda$ the $\mathrm{X}$-ray wavelength measured in nanometres and $2 \theta$ the Bragg angle (in radians). An intermediate value of the Scherrer constant of 0.9 was used in although this may deviate significantly from this value for some crystal shapes [7]. The instrument mediated peak broadening as a function of scattering angle was determined by the linear interpolation between instrument broadening measured for large crystal silicon which produces diffraction peaks at $28.3^{\circ}$ and $47.2^{\circ}$ neglecting possible non-linearity of diffraction profiles with Bragg angle. The Scherrer analysis demonstrated that the crystal axes corresponding to the most intense diffraction peaks were in the $20-50 \mathrm{~nm}$ range.

Nanocomposites were formed using a solvent evaporation method with prior attritor milling in acetone for 4 hours to reduce the TCP particle size. The material was formed into nominally $900 \mu \mathrm{m}$ thick sheets by compression molding. Specimens in the form of disks of diameter $8 \mathrm{~mm}$ were cut from the sheet using a circular punch. The high diameter to thickness aspect ratio was chosen to limit edge effects in order that material swelling and water absorption phenomena would primarily occur along the disk axis to facilitate degradation phenomena assessment in one dimension only.

Ceramic particle sizes in the composites were measured by Wilberforce et al. [8] using a cold cathode field emission electron SEM (S-5500, Hitachi, Japan). Samples differed from those studied in this investigation in that injection molding rather than compression molding was used in specimen manufacture. The modal particle sizes for 10, 20 and $30 \mathrm{wt} \%$ composites is below $150 \mathrm{~nm}$ with relatively few particles exhibiting dimensions greater than $500 \mathrm{~nm}$. Agglomeration and a significantly greater particle size dispersion is observed for $40 \mathrm{wt} \%$ composites which also possess a significant percentage of particles with dimensions greater than $500 \mathrm{~nm}$. Nonetheless, the distribution indicates that a nanocomposite and not a microcomposite is formed using the attritor milling and compression molding procedure.

Specimens manufactured for the tetracycline drug release study were made using the same solvent evaporation technique detailed above with the sole exception that approximately $0.2 \mathrm{~g}$ of tetracycline per $20 \mathrm{~g}$ of composite was added to the slurry 30 minutes prior to completion of attrition milling. 


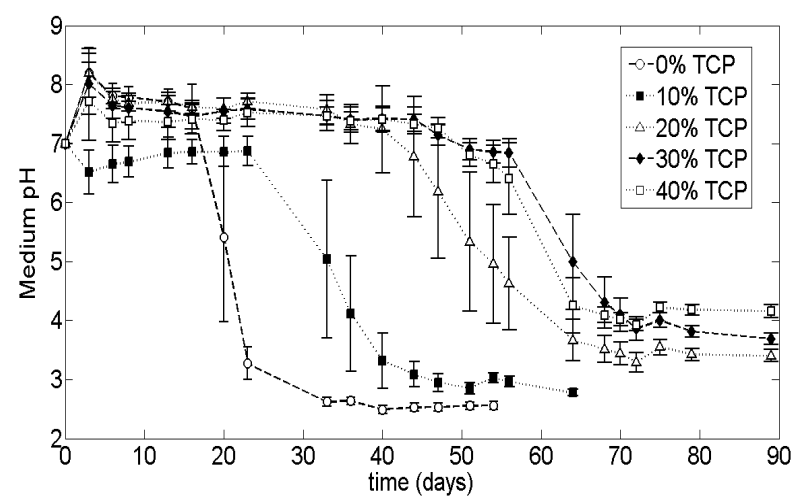

(a)

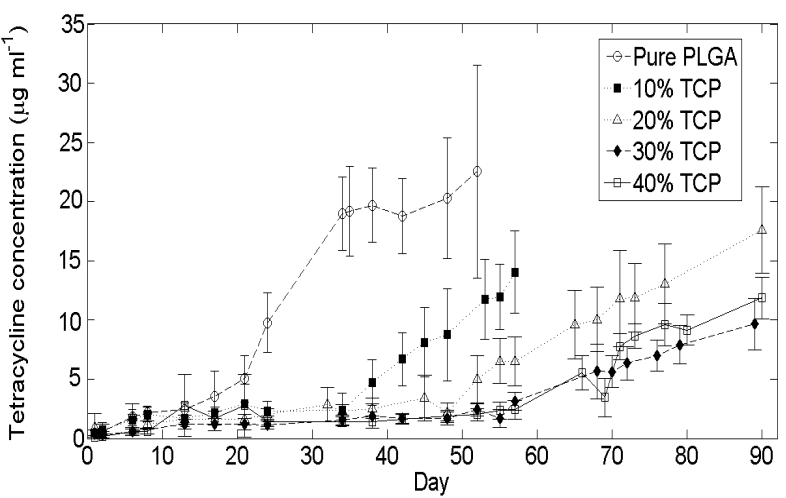

(b)

Figure 1: (a) Aqueous degradation medium $\mathrm{pH}$ and (b) buffer medium tetracycline concentrations versus degradation time for pure PLGA, 10, 20, 30 and $40 \mathrm{wt} \% \alpha \mathrm{TCP}$ nanocomposites showing averaged values for each composite type. .

\subsection{Degradation study}

Samples of each composition were degraded in $40 \mathrm{ml}$ distilled, deionized water (sample to water volume ratio 1:900) at $37.4^{\circ} \mathrm{C}$ in PTFE containers for various times in order to assess composite morphology as functions of degradation time.

Aqueous medium acidity measurements on 27 samples for each composite type were performed at regular intervals using an electronic $\mathrm{pH}$ meter (HI-98230, HANNA Instruments) which was calibrated prior to each testing series using standard solutions. The variation between specimens was determined to be greater than individual time point measurement errors, therefore the uncertainty associated with each material at each time point was calculated as the standard deviation between specimens of the same composition.

The degradation release profiles of the pharmaceutic tetracycline were evaluated for 27 samples of each composite type using a UV spectrometer (Permer-Elkin) calibrated using tetracycline peak absorption at $273 \mathrm{~nm}$ to measure the drug concentration as a function of degradation time. Possible concerns regarding calcium ions influencing tetracycline measurements were evaluated by assessing the effect of known concentrations of aqueous calcium hydroxide on the tetracycline chromophor; no variations in measured tetracycline concentrations with increasing calcium concentration were observed.

Density measurements were performed using a water buoyancy method calculating the specimen bulk density according to

$$
\rho=\rho_{0} \frac{m_{\text {air }}}{m_{\text {air }}-m_{\text {water }}} .
$$

At regular intervals specimens were removed from the degradation study and the mass measured in water, $m_{\text {water }}$, using a modified balance simultaneously recording the water temperature using a thermometer from which the water density, $\rho_{0}$, was determined from a look- up table. Specimens were then dabbed dry and mass in air, $m_{\text {air }}$, measured. The number of specimens per material composition in the density analysis varied considerably between time points. Initially, approximately 20 samples per composite type were measured prior to the start of degradation. This number gradually decreased as the degradation study proceeded as specimens were removed to be characterized destructively using other techniques or the specimens disintegrated during measurement of the density. This reduced the total number of specimens at later time points reduced to approximately 6 for all composite types.

\section{Tomographic analysis}

The X-ray microtomographer used in this investigation is a SkyScan-1072 (SkyScan, Aartselaar, Belgium). Xrays are generated using a Hamamatsu L7 901-01 tungsten target transmission tube possessing a $150 \mu \mathrm{m}$ thick Beryllium window and an incident electron angle of $39^{\circ}$. The generator uses micro-focusing to produce a $8 \mu \mathrm{m}$ diameter X-ray spot size. Depending on the incident electron energy, the $\mathrm{X}$-ray spectrum consists of characteristic $\mathrm{X}$-ray emission and bremsstrahlung caused by electron scattering in the anode. The electron beam may be accelerated through $20-100 \mathrm{kV}$ over the current range of $0-100 \mu \mathrm{A}$ up to a maximum power of $10 \mathrm{~W}$.

The conical X-ray beam geometry permits geometrical magnification achieved by altering the source-sample distance to subtend a larger shadow image at the cost of an increasingly divergent beam through the sample at higher magnifications. Subtended shadow images must be maintained within the camera field of view which is a two-dimensional X-ray CCD camera with a $1024 \times 1024$ pixel resolution 12-bit dynamic range sensor (fibre optic coupling, 3.7:1 image reduction). Conversion of the Xray beam to visible light is achieved using a $22 \mu \mathrm{m}$ thick gadolinium oxysulphide scintillator which is placed over 
the photodetector. Also incorporated are physical beam hardening filters $(0.5 \mathrm{~mm}$ and $1 \mathrm{~mm}$ thick aluminium sheets) which can be imposed between the source and the specimen.

The limited CCD camera spatial extent to record shadow image projections required the specimens to be reduced in size prior to placement in the tomographer specimen chamber such that the shadow image projection remained in the field of view at all times. This was achieved by sectioning the specimens after their removal from the degradation medium and embedding the degraded composite in epoxy resin (Struers Epofix) which was left to cure for 12 hours. After sectioning, exposure of the transect to air led to rapid drying prior to tomographic image acquisition hence all tomographs presented herein represent dehydrated materials.

For all specimens investigated, the source-sample distance was maintained at $52.0 \mathrm{~mm}$ producing a $60 \times$ geometric magnification factor corresponding to a voxel size of $4.67 \mu \mathrm{m}$. This magnification was considered adequate to identify features of interest but not so high as to incur disproportionate penumbral blurring.

Shadow images were acquired over a full $360^{\circ}$ stage rotation using rotation steps of $0.45^{\circ}$. Complete $360^{\circ}$ rotation rather than $180^{\circ}$ was used to minimize possible "star artifacts" which are caused by high attenuation coefficient contrasts between adjacent phases.

Intensity differences in the illuminating source or inefficiencies in individual CCD pixel element detection sensitivity can cause errors in measurements of transmitted $\mathrm{X}$-ray intensities which yield reconstruction image artefacts [9]. Correction involves the acquisition of a dark field image (no incident X-rays) which is used as the reference intensity for each pixel, both for measurement of the X-ray intensity recorded at the photodetector after traversing the sample, $I$, and the intensity incident on the sample, $I_{0}$.

Beam hardening in the sample was reduced by the use of a $1 \mathrm{~mm}$ aluminum filter to preferentially absorb lowerenergy X-rays producing a more monochromatic beam at the cost of a decreased count rate and greater signalto-noise ratio. The tomographic reconstruction software NRECON does permit the application of a beamhardening correction which fits a polynomial as a function of the path length through the material which requires a greater number of terms for highly attenuating materials. This function was not utilized due to the reduced signal to noise ratio caused by the correction and difficulties in applying the technique to inhomogeneous materials which may suffer from the subjectivity of correction validation by the empiricist.

The percentage error on the pixel count-rate, $\sigma$, is equal to the inverse square-root of the number of counts

$$
\sigma=\frac{1}{\sqrt{N}}
$$

Minimization of $\sigma$ can achieved by increasing the acquisi- tion times for each rotation angle either by extending the dwell time, recording multiple shadow images for each step and averaging or a combination of the two. The reductions in count rate by highly attenuating materials such as high TCP content nanocomposites requires longer acquisition times to improve shadow image signal to noise ratio. In manipulating the dwell time it must be noted that it is not the count rate which is detected but the total number of counts. The ramifications for this difference is that saturation may occur in CCD elements if the total number of counts is too high which requires verification during set-up. Frame averaging was therefore preferred to improve the image quality.

Tomographic reconstruction was performed using the SkyScan NRecon package supplied by the manufacturer which uses the Feldkamp cone-beam algorithm reconstruction method to account for the X-ray source conical geometry [10]. Reconstruction was performed for each CCD pixel row which produces a stack of 1024 tomographs. However, the upper and lower pixel rows are compromised by the cone-beam geometry therefore the default reconstruction stack range excludes the upper and lower CCD elements from reconstruction unless over-ridden by the experimenter.

Prior to reconstruction, post-alignment was performed in NRECON to correct possible rotation axis misalignment during acquisition which can cause blurring or doubling in reconstructed images. Correction values are obtained by comparing pseudo-parallel projections of conebeam shadow images at $0^{\circ}$ and $180^{\circ}$ and calculating the misalignment value to achieve optimal image convergence. Tomographic reconstructions were outputted as 256 grey-scale 8 Mbit BMP images.

Tomographic image analysis was performed using ImageJ [11]. For all specimens tested, sample inclination an deviation from the vertical exhibited a negligible effect on measured distances. The maximum registered deviation from the vertical across all samples measured was $6.5^{\circ}$; typical deviations ranged from $0.5-4^{\circ}$ which leads to a distance error of less than $1 \%$ which is negligible.

Warping of the sample can cause the plane vector for each tomograph to deviate from the vertical at different stack heights. Image alignment was performed using the ImageJ application "TurboReg" developed by Thevenaz et al. [12] using the translation aliasing mode in which straight lines are mapped to straight lines in the target image of identical operation conserving the distance between point pairs of the form $\mathrm{x}=\mathrm{u}+\Delta \mathrm{u}$.

Prior to tomographic evaluation, the resin used to embed the specimens prior to sectioning were removed digitally using Adobe Photoshop(R. 


\begin{tabular}{cccc}
\hline Material & Final pH & $\begin{array}{c}\text { Acidification } \\
\text { onset (days) }\end{array}$ & $\begin{array}{c}\text { Tetracycline } \\
\text { release } \\
\text { onset (days) }\end{array}$ \\
\hline \hline PLGA & 2.5 & 15 & 15 \\
$10 \%$ & 2.9 & 22 & 33 \\
$20 \%$ & 3.3 & 32 & 45 \\
$30 \%$ & 3.6 & 43 & 55 \\
$40 \%$ & 4.2 & 43 & 55 \\
\hline
\end{tabular}

Table 1: PLGA(50:50) and PMNC degradation study information showing final aqueous medium acidity at the end of composite degradation, time at start of significant medium acidification and tetracycline release onset times.

\section{Results}

Degradation medium acidification onset is an indicator of oligomer release from the polymer matrix. According to figure 1a therefore, mass loss from the material does not occur until approximately 40 days into the degradation process for $20 \mathrm{wt} \%$ and higher ceramic loading nanocomposites. The equivalent erosion onset time for $10 \mathrm{wt} \%$ composite is approximately 25 days post immersion.

The delay in $\mathrm{pH}$ change initiation with increasing $\alpha$ TCP content is particularly apparent for pure PLGA, $10 \mathrm{wt} \%$ and $20 \mathrm{wt} \%$ composites. The convergence of $30 \mathrm{wt} \%$ and $40 \mathrm{wt} \%$ behaviour indicates that autocatalyst saturation occurs around $30 \mathrm{wt} \%$ which corroborates previous empirical studies of this material by Yang et al. [2] and Ehrenfried et al. [3].

Tetracycline release data are presented in figure $1 \mathrm{~b}$. Tetracycline is highly insoluble in water hence detection in the buffer medium is primarily attributable to polymer erosion which releases the pharmaceutic. The initial increase in detected tetracycline concentrations at early degradation times is most likely surface release. The start of tetracycline release is closely correlated with medium acidification and becomes progressively later for higher ceramic loaded nanocomposites which is a consequence of autocatalysis inhibition. Behavioural convergence is also observed in the release profiles of 30 and $40 \mathrm{wt} \%$ nanocomposites in an analogous pattern to buffer medium acidity which is caused by saturation of the autocatalysis neutralization effect.

A summary of final degradation medium $\mathrm{pH}$, onset of significant medium acidification and initialization of tetracycline release are presented in table 1 .

Figure 2a presents the bulk density evolution for nanocomposites and pure PLGA. Included for each nanocomposite type are curves of best-fit according to

$$
\rho(t)=A+B t^{\frac{1}{2}}
$$

where $A$ and $B$ represent fitting parameters. The $\sqrt{t}$ dependence of nanocomposite density evolution is suggestive of a diffusion controlled process. The fit quality of equation 5 for $10 \mathrm{wt} \%$ and $20 \mathrm{wt} \%$ composites begins to deviate from a $\sqrt{t}$ dependence at around 25 days after the start of degradation. This is most likely attributable to the increasing importance of polymer hydrolysis and mass loss as indicated by drug release and medium acidification which occurs around this time. The $\sqrt{t}$ trend is followed for $30 \mathrm{wt} \%$ and $40 \mathrm{wt} \%$ over the whole of the recorded data set which is expected due to ceramic inhibition of autocatalytic degradation until late degradation times.

Figure 2 also demonstrates that the PLGA density evolution is not well described by equation 5 . This indicates that different processes occur in the pure polymer compared with the nanocomposites which is discussed further in section 5 .

Normalized composite masses measured in air according to figure $2 \mathrm{~b}$ demonstrate that the mass of $10 \mathrm{wt} \%$ and $20 \mathrm{wt} \%$ samples doubles in approximately 13 days. The equivalent doubling time for the $30 \mathrm{wt} \%$ and $40 \mathrm{wt} \%$ composites is 35-40 days. This increase in mass is a direct consequence of water imbibition into the composite. Evaluation of the normalized mass per unit volume of polymer shows that mass increase is negligible in pure PLGA compared with the nanocomposites suggestive of different water sorption kinetics between the pure polymer and nanocomposites.

An example of the morphological features exhibited by the materials in X-ray tomographs is presented in figure 3 which shows the direction of water diffusion evaluated assuming infinite planar geometry. Regions of reduced attenuation coefficients are termed the "periphery" and the central part which exhibits higher attenuation coefficients is defined as the "core". Frequently observable in the core regions are particles exhibiting higher attenuation coefficients above the mean which are most likely clusters of ceramic particles not broken down by attritor milling during sample fabrication.

Examples of tomographic montages for $30 \mathrm{wt} \%$ nanocomposites are presented for degradation times of 7,21 and 42 days in figure 4 in which specimens are oriented such that water penetration occurs in the horizontal direction.

Regions of lower attenuation coefficient are observable on the lateral portions of each material with clear advancement towards the centre occurring between the time points. Void formation in the periphery is observable in the more degraded materials which absent in tomographs of the same material degraded for 7 days.

A significant problem with material behavioural analysis based on individual tomographs as per figure 4 is the arbitrary nature of the tomographs selected by the user; typically 900 individual tomograph slices were re- 


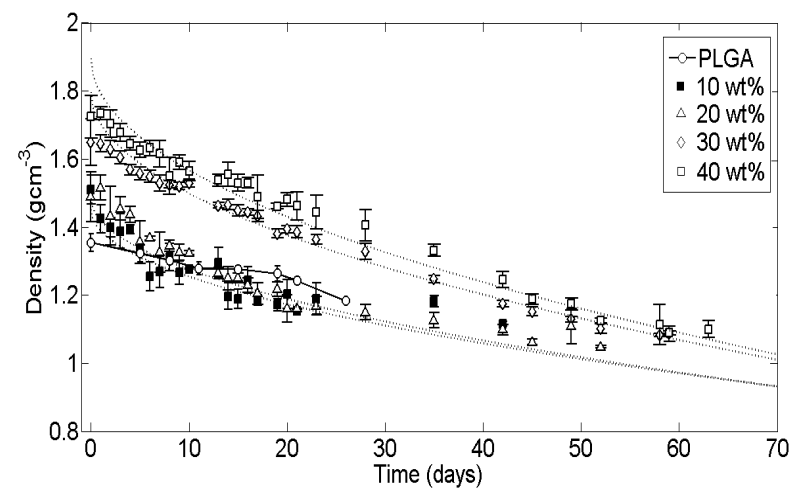

(a)

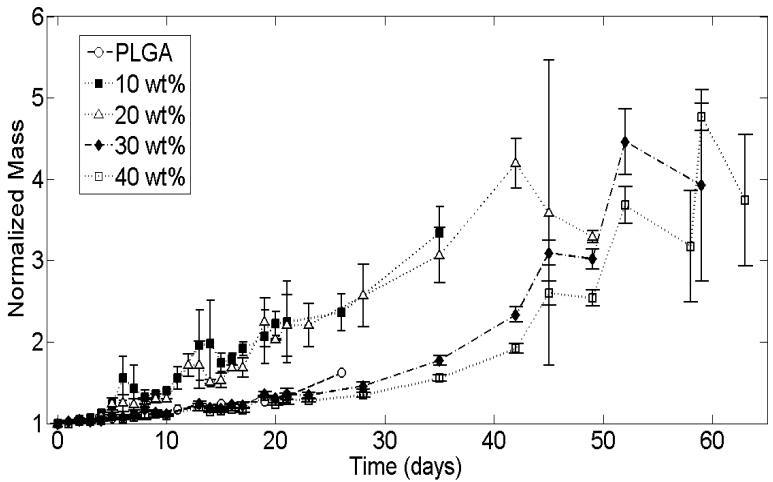

(b)

Figure 2: (a) Densities of pure PLGA(50:50) specimens and nanocomposites measured using a buoyancy method versus degradation time, nanocomposite density versus time is fitted with a $t^{\frac{1}{2}}$ function described in section 4 . (b) Normalized average sample mass with respect to undegraded sample at start of degradation measured in air

constructed for each specimen. A more impartial method of assessing the effects of degradation on material structures is to calculate the average properties over the entire stack using the median stack projection function in ImageJ. A disadvantage of stack median projection is that random pore formation in the peripheral region is obscured.

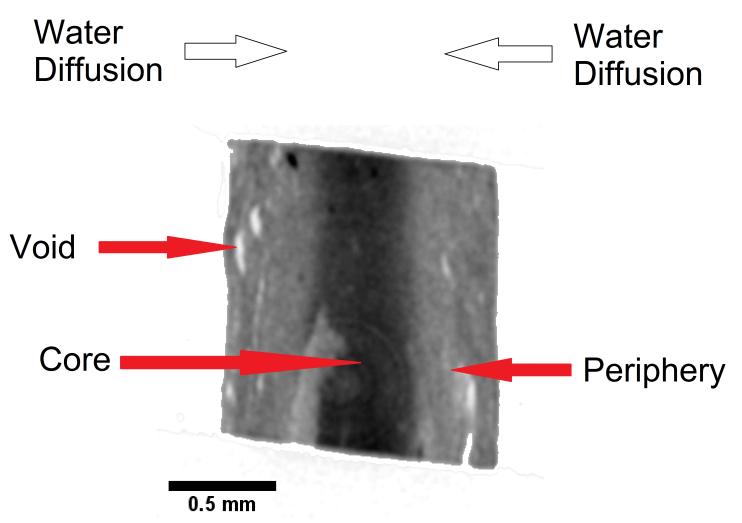

Figure 3: Definitions of core and peripheral regions observed in tomographs of $30 \mathrm{wt} \%$ nanocomposite degraded for 21 days. Also indicated are voids formed during degradation and the water diffusion direction.

Attenuation coefficient transects determined from median projection images for each composite type at varying degradation times is shown in figure 5 . Tomographic analysis of PLGA was attempted but the material's low stiffness and rapid degradation did not permit tomographic image acquisition. Transect attenuation coefficient profiles show that the core region's attenuation coefficient (which is proportional to material density) decreases progressively as degradation proceeds. The attenuation coefficient increase at the specimen edge is probably a beam-hardening artefact.
The reduced attenuation coefficient for the periphery is either caused by TCP diffusion out of the material or composite swelling and consequent density reduction. Yang et al. [2] showed that $\alpha \mathrm{TCP}$ release from the matrix evaluated using calcium ion concentrations is absent during the first 25 days post immersion for $10 \mathrm{wt} \%$ nano$\alpha$ TCP composites which increases to approximately 40 days for $20 \mathrm{wt} \%$ composites and higher ceramic loaded composites. This eliminates the loss of TCP from the matrix as the cause of the attenuation coefficient reduction. Direct quantitative comparison between this study and that of Yang is not possible due to the use of deionized, distilled water as the degradation medium in this investigation as opposed to phosphate buffered saline solution. Other variables measured by Yang such as degradation medium acidification are closely correlated with equivalent measurements performed in this investigation. Therefore, the attenuation coefficient reduction must be attributable to material swelling.

\subsection{Measurement uncertainties}

\section{Analysis}

PLGA is a bulk eroding polymer therefore it is expected that polymer degradation occurs faster in the sample interior due to autocatalysis producing a lozenge-like structure. For all but one sample investigated, the attenuation coefficient of the central region never decreases below that of the periphery. The sole exception is a $40 \mathrm{wt} \%$ nanocomposite after degrading for 49 days (see figure 5) which may exhibit central hollow formation due to autocatalyst accumulation and accelerated degradation.

The total transect width for each nanocomposite material studied is plotted in figure 6a. Included on the plot is a curve of best-fit which describes the composite 


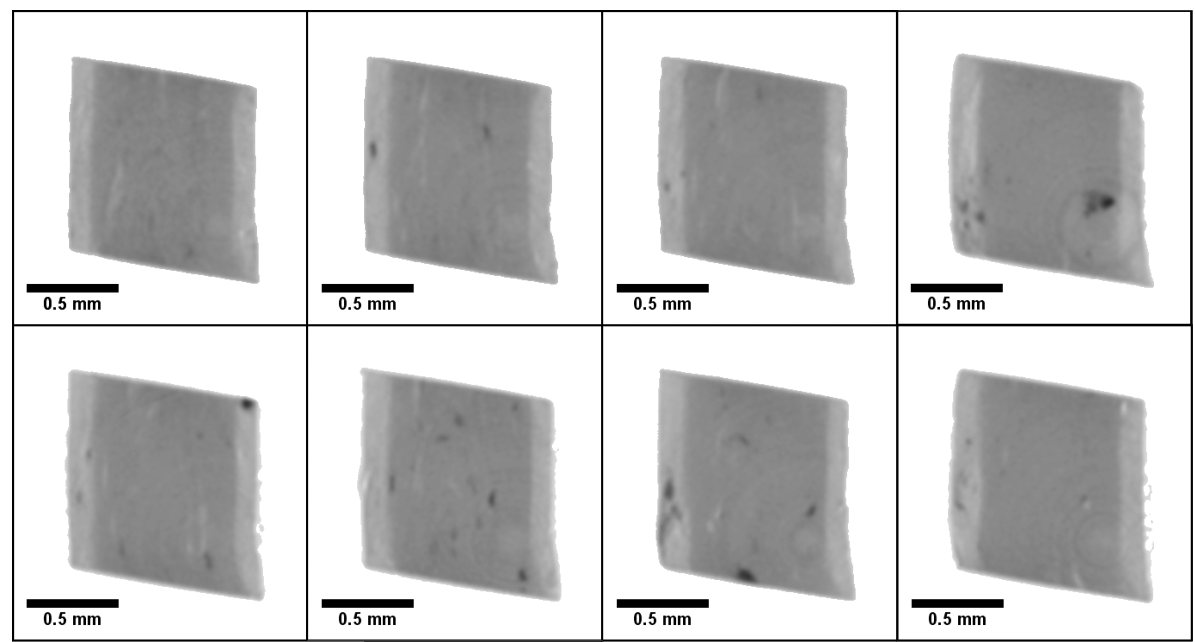

(a)

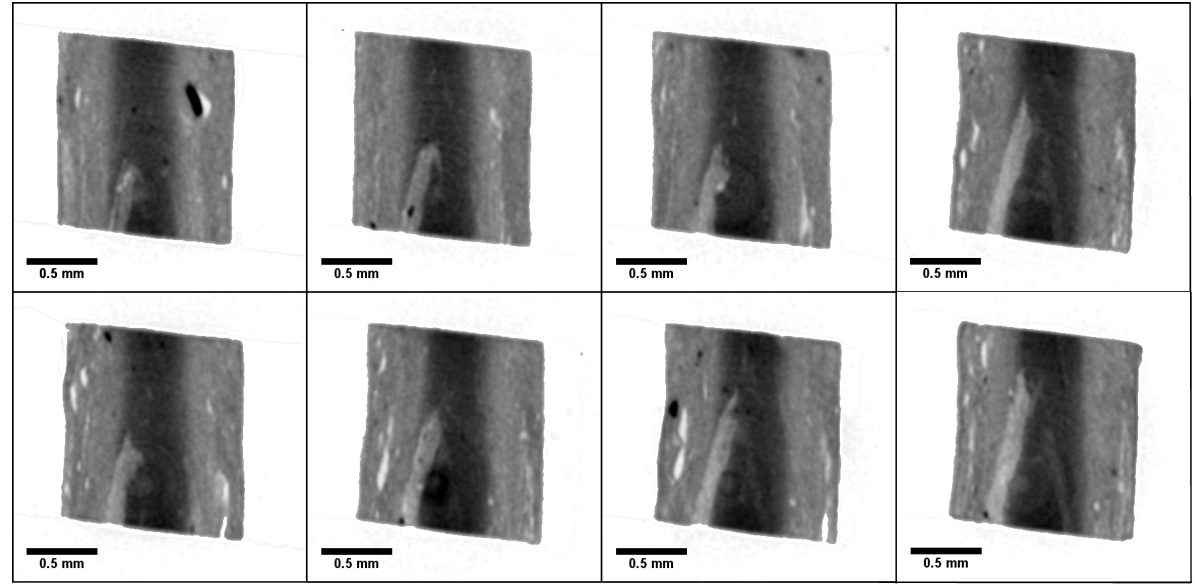

(b)

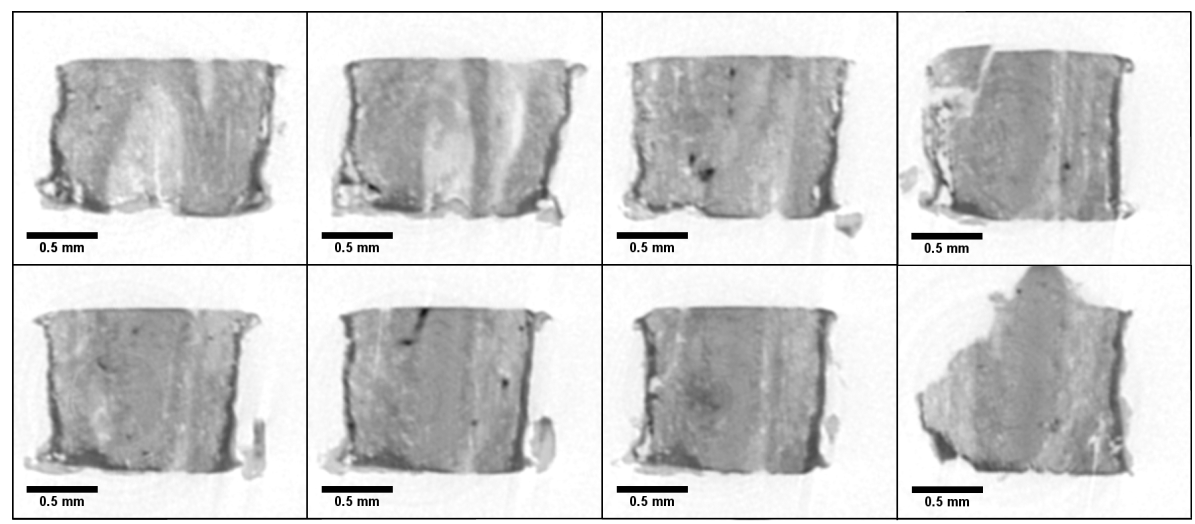

(c)

Figure 4: X-ray microtomographs of $30 \mathrm{wt} \%$ TCP nanocomposites at varying stack positions degraded for a) 7 days b) 21 days and c) 28 days. The reader should note that differing greyscale normalization criteria have been employed between the time points which does not enable quantitative attenuation coefficient comparisons. 

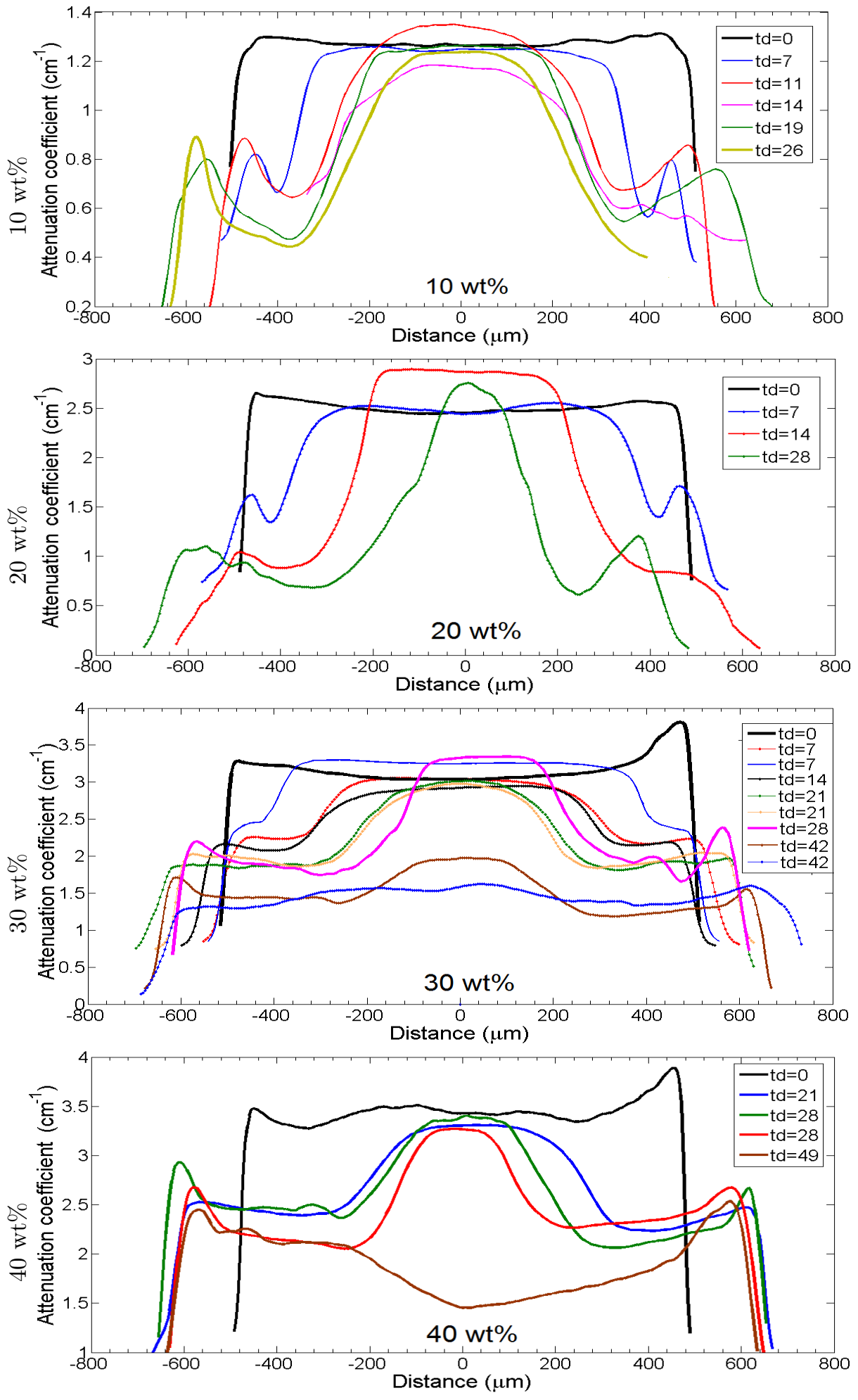

Figure 5: Average attenuation coefficient variation across transects of 10, 2030 and 40 wt\% nanocomposites for various degradation times acquired using an accelerating voltage of $60 \mathrm{kV}$. 
transect width according to

$$
\tau(t)=A+B \operatorname{erfc}(a t+c) .
$$

fitted using GNUPLOT which yields $A=1021 \pm 14 \mu \mathrm{m}$, $B=147 \pm 9 \mu \mathrm{m}, a=-0.11 \pm 0.02$ days $^{-1}$ and $c=1.66 \pm$ 0.35 days $^{-1}$. The curve of best-fit possesses a reduced $\chi^{2}$ of 2.0 for 19 degrees of freedom.

Assuming that the reduction in specimen density observed in microtomographic data is caused by Fickian water diffusion neglecting possible ceramic leeching, omitting edge effects for a plane sheet of thickness $l$, and using the symmetry of the problem the position of the mid-point of the transitional region may be expressed as

$$
\frac{C(x, t)}{C_{0}}=\operatorname{erfc}\left(\frac{\frac{l}{2}+x}{2 \sqrt{D t}}\right)=\frac{1}{2}
$$

where $D, t$ and $x$ represent the water diffusion coefficient, immersion time and the transect distance defined in relation to the sample centre line. The distance from the sample centre line, $x$ is equal to half the core region thickness i.e. $x=\frac{\tau}{2}$. Therefore, using the Taylor series expansion of the error function

$$
\operatorname{erfc}(z)=1-\frac{2}{\sqrt{\pi}}\left(z-\frac{z^{3}}{3}+\frac{z^{5}}{10}-\frac{z^{7}}{42}+\ldots\right)
$$

neglecting higher order terms in $z$, equation 7 may be expressed as

$$
\sqrt{\pi D t}=l+\tau .
$$

The core width, $\tau$ was defined using a core boundary criterion which was defined as mid-point of the transition between core and non-core in the median tomographic image projections presented in figure 5 . The result of fitting $\tau$ as a function of $t^{\frac{1}{2}}$ according to equation 9 is shown in figure $6 \mathrm{~b}$. The curve is a line of best fit with gradient $B=-0.038 \pm 0.002 \mathrm{~m} \cdot \mathrm{s}^{-\frac{1}{2}}$ and intercept $A=1012 \pm 32 \mu \mathrm{m}$ corresponding to an effective diffusion coefficient of $D=6.1 \times 10^{-14} \mathrm{~cm}^{2} \mathrm{~s}^{-1}$. The quality of the fit is poor (a $\chi^{2}$ of 6.7 for 21 degrees of freedom) which is caused by deviations from the $t^{\frac{1}{2}}$ dependence for core region dimensions at advanced degradation times which is probably caused by material degradation.

A schematic of the average degradation profile of the nanocomposites with time showing average specimen swelling and core region thickness behaviour is presented in figure 7 . The swelling complementary error function time dependence and the $t^{\frac{1}{2}}$ core region thickness dependence suggest that the core-periphery structure is controlled by Fickian diffusion during the initial degradation phase. However, a $t^{\frac{1}{2}}$ dependence is a necessary but not sufficient criterion to establish linear diffusion as the cause of the core-peripheral structure.
The relatively abrupt transition of the attenuation coefficient from core to periphery as demonstrated in attenuation coefficient profiles in figure 5 appears inconsistent with a diffusion controlled process. It is possible that the front formation is representative of a water or degradation induced polymer phase change from a glassy to rubbery state which requires further investigation.

An additional indication that the attenuation coefficient behavioural evolution might be attributable to a phase change rather than diffusion is the specimen transect width and core thickness behaviour (figures $6 \mathrm{a}$ and $6 \mathrm{~b})$. Both the specimen transect width and the core thickness exhibits no variation with nanocomposite ceramic content. This contrasts with the inverse relationship between ceramic volume fractions and water diffusion coefficients for HA-Poly(3-hydroxybutyrate-co3-hydroxyvalerate) nanocomposites incorporating a silane coupling agent measured by Tang et al. [13] using water imbibition measurements assuming Fickian diffusion. This inverse relationship between ceramic filler content and water diffusion coefficient is expected due to the increase in the tortuosity of diffusion paths caused by the presence of the impermeable HA crystals.

The range of ceramic volume filler fractions assessed by Tang et al. [13] was 0 to 0.3 which is comparable to the ceramic volume fractions in the $\alpha$ TCP-PLGA nanocomposites in this study which is 0 to 0.216 . The water uptake in PHBHV nanocomposites saturated approximately 3 days post immersion in water yielding water diffusion coefficients for the materials measured at $37^{\circ} \mathrm{C}$ for $\mathrm{PHBHV}$ in the $6.01 \times 10^{-9}$ to $14.11 \times 10^{-9} \mathrm{~cm}^{2} \mathrm{~s}^{-1}$ range.

An equivalent assessment of water absorption characteristics in PLGA- $\alpha$ TCP composites using similar principles to Tang et al. [13] is not possible in this investigation due to the inability to define the mass of water in specimens at equilibrium $\left(M_{\infty}\right)$. Bulk density and normalized mass measurements according to figure 2 show that, $M_{\infty}$ is not attained for $10 \mathrm{wt} \%$ and $20 \mathrm{wt} \%$ composites until around $t_{d}=35$ during which time significant polymer hydrolysis has occurred. Future assessment of sorption behaviour requires the fabrication of much thinner samples such that the time required for complete specimen saturation with water be significantly less that the characteristic polymer degradation time allowing polymer matrix changes to be neglected. 


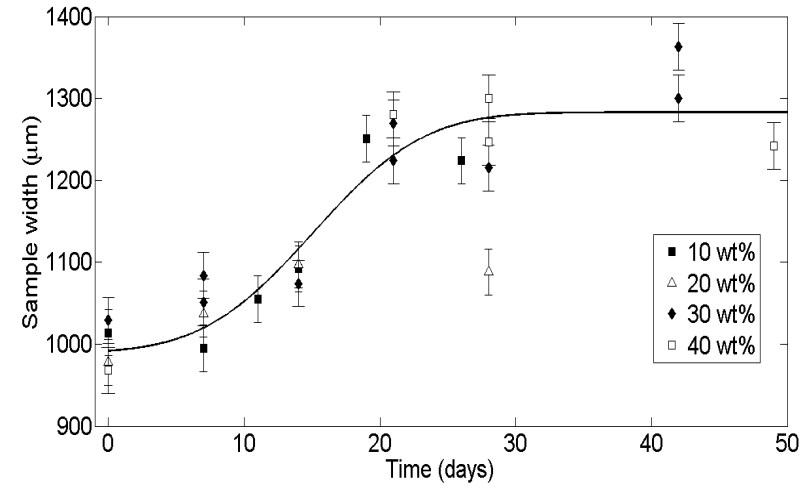

(a)

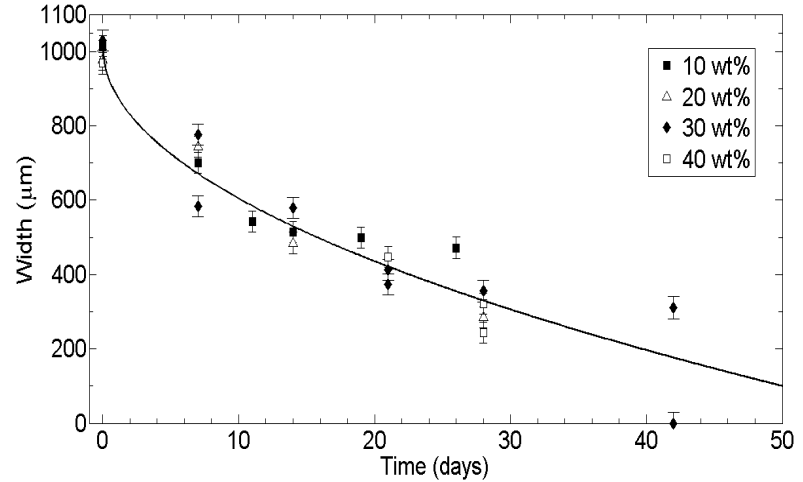

(b)

Figure 6: (a) Composite transect width as function of degradation time measured using X $\mu \mathrm{T}$ with curve of best fit. (b) Core region width versus degradation time with curve of best-fit.

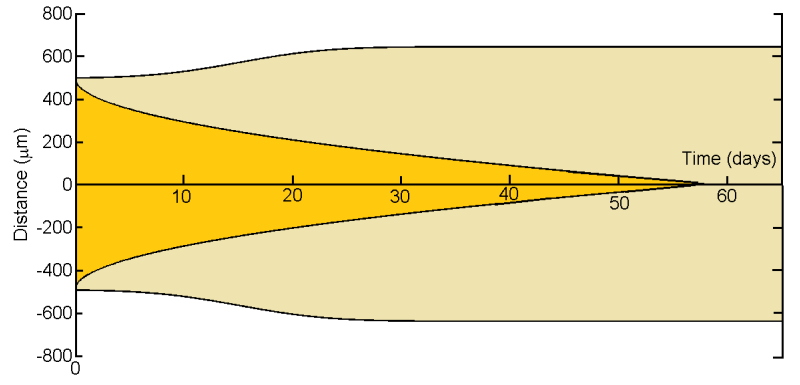

Figure 7: Evolution of nanocomposite swelling and hydration front behaviour. The vertical axis denotes the position of the material edge (outermost curve) and the core region boundary (inner curve) with respect to the sample meridian.

Front behaviour as a result of hydration has also been observed during the degradation of polyglycolic acid (PGA) which is a semi-crystalline polymer. In a series of studies by Hurrell et al. [14, 15, 16], front formation at the material surface was documented after a latent period of $7 \pm 2$ days post immersion in an aqueous medium. Subsequently, the fronts advanced at a rate of $0.033 \pm 0.002 \mathrm{~mm} /$ day towards the sample centre. Plasticization was discounted as the origin of the front which based on the diffusion coefficient of water in PGA $\left(D_{\mathrm{H}_{2} \mathrm{O}}=5 \pm 1 \times 10^{-9} \mathrm{~cm}^{2} \mathrm{~s}^{-1}[17]\right)$ and the material dimensions which results in complete water saturation and hence plasticization of the polymer 6 hours postimmersion.

Front formation and movement in PGA were described in terms of a four-stage reaction-erosion model [18] in which the first stage, completed several hours after immersion, consists of rapid water imbibition and polymer plasticization. In situ polymer de-esterification defines the second stage where oligomers are too large to be mobile and hence are unable to diffuse into the buffer solution. The third stage begins when oligomers near the surface are sufficiently small to diffuse out of the sample; the resulting polymer erosion and further water imbibition causes the formation of a moving front which characterizes the stage. Elimination of the water gradient occurs when the fronts converge which is defined as the fourth and final stage of the reaction-erosion model.

The linear nature of the front progression and latent formation period for PGA contrasts with the $t^{\frac{1}{2}}$ dependence for $\alpha$ TCP-PLGA nanocomposites in which swelling and front advance occurs immediately after immersion in the degradation medium.

In summary, the non-inverse relationship between ceramic filler content and apparent diffusion coefficient measured for $\alpha$ TCP-PLGA nanocomposites may be due to a polymer phase change and not water diffusion controlling the internal specimen morphology. Additionally, a threshold ceramic content may exists above which further nanoparticle contribution to the front behaviour is negligible. Although $\mathrm{X} \mu \mathrm{T}$ for pure PLGA does not exist, the density evolution difference observable in 2a between pure PLGA and the nanocomposites is indicative of differing material dynamics caused by $\alpha \mathrm{TCP}$ nanoparticle addition.

\section{Conclusion}

This paper describes the evolution of the morphology of PLGA(50:50) and nanocomposites formed from PLGA(50:50) and nanoparticulate $\alpha \mathrm{TCP}$ in various weight loadings as a function of degradation time in an aqueous medium. Further information regarding degradation kinetics was achieved by assessing changes in degradation medium acidity, specimen density and pharmaceutic release.

$\mathrm{X}$-ray microtomography demonstrates that morphological changes occurring during material degradation is attributable to hydration which exhibits a preponder- 
ance over de-esterification during the initial degradation phase. Material expansion follows an error function of time and the internal front advance is well described by a $t^{\frac{1}{2}}$ time dependence which are indicative of a Fickian diffusion controlled process. Nonetheless, these are necessary but not sufficient conditions for linear diffusion and further work is required to establish whether this is indeed caused by Fickian diffusion or whether the observed front is caused by a water and polymer degradation effected phase changes in the polymer from a glassy to a rubbery state.

\section{Acknowledgements}

The authors would like to thank Miss Camille Flament for assistance in specimen preparation. This work was supported by the UK EPSRC under grants $\mathrm{EP} / \mathrm{P} 502365 / 1$ and $\mathrm{EP} / \mathrm{P} 504120 / 1$.

\section{References}

[1] C.E Barrett, R.E. Cameron, S.E. Best, Bioceramic and Biopolymer Nanocomposite Materials for Use in Orthopaedic Applications CRC Press, Taylor and Francis (2012)

[2] Z. Yang, S.M. Best, R.E. Cameron, The influence of a -tricalcium phosphate nanoparticles and microparticles on the degradation of poly(D, L-lactideco-glycolide), Advanced Materials 21 (2009) 3900

[3] L.M. Ehrenfried, D. Farrar, D. Morsley, R.E. Cameron, Mechanical behaviour of interpenetrating co-continuous $\beta$ - TCP-PDLLA composites, Bioceramics 20 (2008) 361

[4] F. Meyer, J. Wardale, S. Best, R. Cameron, N. Rushton, R. Brooks, Effects of lactic acid and glycolic acid on human osteoblasts: A way to understand PLGA involvement in PLGA/calcium phosphate composite failure, Journal of Orthopaedic Research, 30 (2012) 864

[5] M. Vert, Polymeric biomaterials: Strategies of the past versus strategies of the future, Progress in Polymer Science, 32 (2007) 755

[6] Y. Chen, S. Zhou, Q. Li, Mathematical modelling of degradation for bulkerosive polymers applications in tissue engineering scaffolds and drug delivery systems, Acta Biomaterialia 7 (2011) 1140

[7] B.D. Culity, S.R. Stock, Elements of X-Ray diffraction, 3rd Ed. Pearson Educational International (2001)

[8] S.I.J. Wilberforce, S.M. Best, R.E. Cameron, J. Mater. Sci.: Materials in Medicine 21 (2010) 3085

[9] E.N. Landis, D. T. Keane, X-ray microtomography, Materials Characterization 61 (2010) 1305

[10] L.A. Feldkamp, L.C. Davis, J.W. Kress, Practical cone-beam algorithm, Journal of the Optical Society of America 1 (1984) 612

[11] C.A. Schneider, W.S. Rasband, K.W. Eliceiri, NIH Image to ImageJ: 25 years of image analysis, Nature Methods 9 (2012) 671

[12] P. Thévenaz, U.E. Ruttimann, M. Unser, A pyramid approach to subpixel registration based on intensity, IEEE Transactions on Image Processing, 7 (1998) 27

[13] C.Y. Tang, D.Z. Chen, T.M. Yue, K.C. Chan C.P. Tsui, P.H.F. Yu, Water absorption and solubility of PHBHV/HA nanocomposites Composites Science and Technology 68 (2008) 1927

[14] S. Hurrell, R. E. Cameron, Polyglycolide: degradation and drug release. Part I: Changes in morphology during degradation, Journal of Materials Science: Materials in Medicine 12 (2001) 811

[15] S. Hurrell, G. E. Milroy, R. E. Cameron, The degradation of polyglycolide in water and deuterium oxide. Part I: The effect of reaction rate, Polymer International 44 (2003) 1421

[16] S. Hurrell, R. E. Cameron, The effect of buffer concentration, $\mathrm{pH}$ and buffer ions on the degradation and drug release from polyglycolide, Polymer International, 52 (2003) 358

[17] G.E. Zaikov, Quantitative aspects of polymer degradation in the living body, Journal of Macromolecular Science, Reviews in Macromolecular Chemistry and Physics C25 (1985) 551

[18] S. Hurrel, G.E. Milroy, R.E. Cameron, Polyglycolide: Degradation and drug release. Part II: Drug release, Journal of Materials Science: Materials in Medicine 14 (2003) 457 